\title{
Electron Holography of Magnetic Vortices in Chains of FeNi Nanoparticles
}

\author{
R.E. Dunin-Borkowski, ${ }^{*}$ M.J. Hÿtch, ${ }^{* *}$ and M.R. Scheinfein***
}

* Department of Materials Science and Metallurgy, University of Cambridge, Pembroke Street, Cambridge CB2 3QZ, United Kingdom

** Centre d'Etudes de Chimie Métallurgique-CNRS, 15 rue G. Urbain, 94407 Vitry-sur-Seine, France

*** Department of Physics, Simon Fraser University, 8888 University Drive, Burnaby, BC V5A 1S6, Canada

The magnetic properties of chains of nanoparticles are of interest both fundamentally and for applications that include data storage. When the sizes of such particles approach the exchange length, their magnetic microstructure may be dominated by surface boundary conditions, external fields and flux closure through neighboring particles, in addition to magnetostatic and exchange energies. Here, we use electron holography to image the magnetic remanent states of chains of single crystalline $\mathrm{Fe}_{0.5} \mathrm{Ni}_{0.5}$ nanoparticles, with an emphasis on understanding the formation of magnetic vortices. The particles were prepared by rapid condensation in a levitation furnace and collected in hexane, where they formed $2-3 \mathrm{~nm}$ oxide shells. The average particle diameter of $50 \mathrm{~nm}$ was close to the critical size for the single-domain to vortex transition in $\mathrm{Fe}_{0.5} \mathrm{Ni}_{0.5}$.

Figure 1a shows a schematic ray diagram that illustrates the formation of an off-axis electron hologram in the field emission gun (FEG) transmission electron microscope (TEM). Holograms of chains of FeNi particles were recorded digitally at $300 \mathrm{kV}$ with the sample in field-free conditions, using a Philips CM300ST FEG TEM that was operated in Lorentz mode with the conventional objective lens switched off. The magnetic remanent states of two chains of FeNi particles are shown in Figs. $1 \mathrm{~b}$ and $\mathrm{c}$ in the form of phase contours, which are overlaid onto chemical maps that show the oxygen distribution in each particle. The mean inner potential contribution to the phase has been removed from each image using a procedure described elsewhere [1]. Figures 1d and e show schematic representations of the magnetic microstructure in the two chains. In Fig. 1b, a magnetic vortex core of large diameter $(23 \pm 2 \mathrm{~nm})$ forms inside a 75 -nm-diameter particle that is sandwiched between two smaller particles. The magnetization in the particle spins around this 'flux tube', as shown schematically in Fig. 1d, and as confirmed using micromagnetic simulations in Fig. 2. The flux tube forms, in part, as a result of the field that the particle experiences from its neighbors in the chain. In contrast, a vortex oriented perpendicular to the chain axis and visible end-on has a smaller diameter of $9 \pm 2 \mathrm{~nm}$, as shown in Fig. 1c for a 71-nm-diameter particle. The height of the step in phase shift across several FeNi particles is shown as a function of particle diameter in Fig. 3. Results that are consistent with the predicted phase shift for a uniformly magnetized, isolated sphere are shown as bold circles. Results from particles containing flux tubes, which are shown as open circles, suggest that the flux tube width is independent of particle size to within experimental error [2].

\section{References}

[1] R.E. Dunin-Borkowski et al., Ultramicroscopy 74 (1998) 61.

[2] This research was supported by the Royal Society and carried out in the framework of the CNRS-funded European Research Group "Quantification and measurement in TEM". 

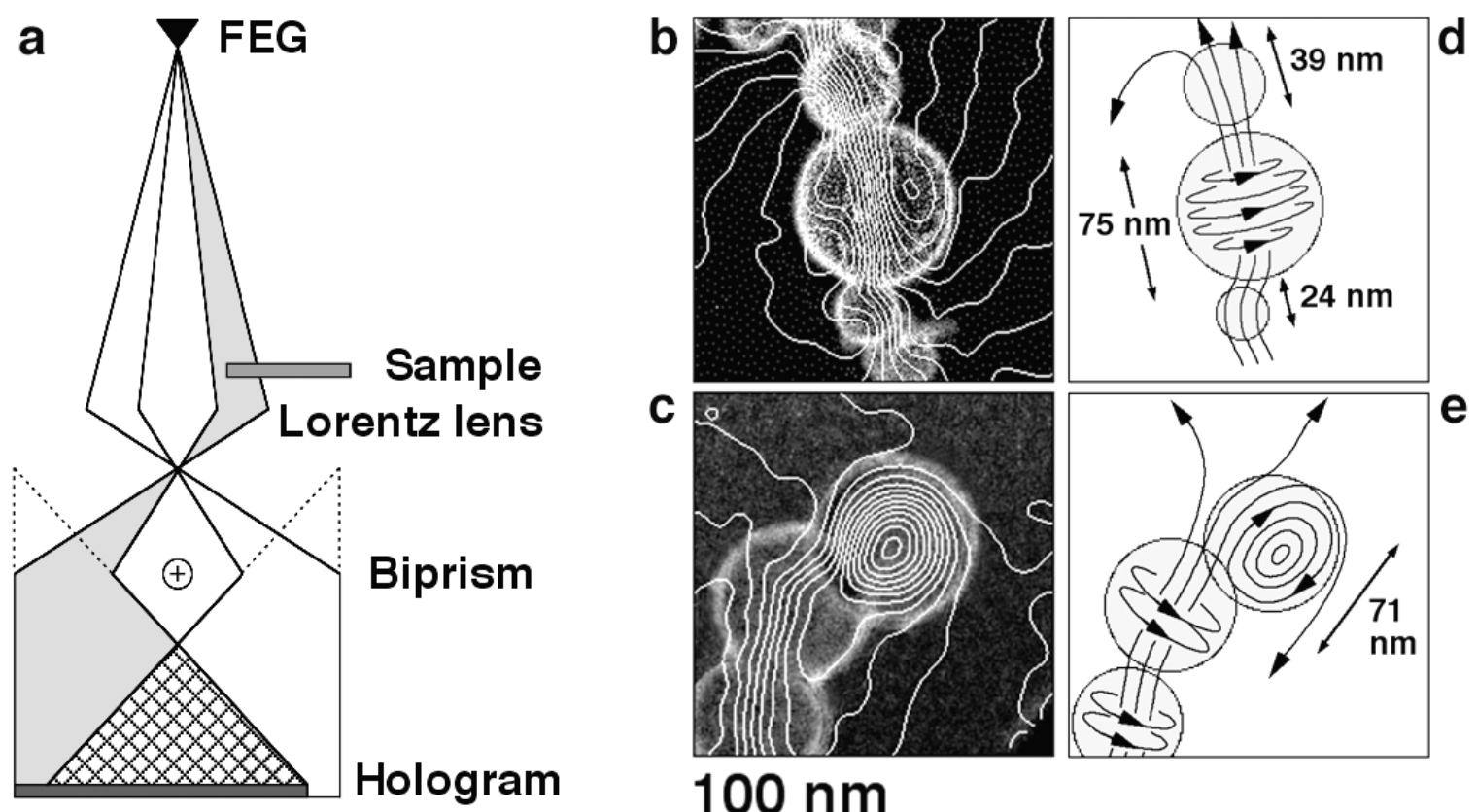

$100 \mathrm{~nm}$

FIG. 1. a) Schematic ray diagram illustrating the formation of an off-axis electron hologram in the field emission gun (FEG) transmission electron microscope. b) and c) Experimental phase contours showing the strength of the local magnetic induction in two different chains of $\mathrm{Fe}_{0.5} \mathrm{Ni}_{0.5}$ particles. The contours, which have been overlaid onto chemical maps of the chains, have spacings of 0.083 and 0.2 radians, respectively. The mean inner potential contribution to the phase has been removed from each image. d) and e) show schematic representations of the magnetic microstructure.

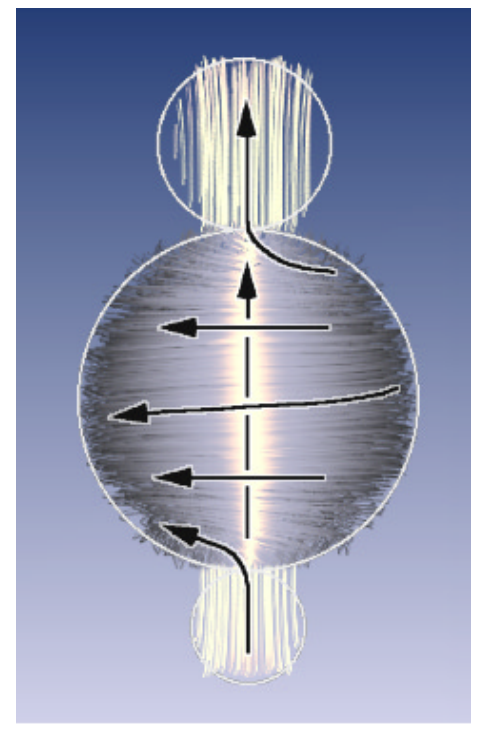

\section{$20 \mathrm{~nm}$}

FIG. 2. Simulated streamlines illustrating the 3 -D vector field in the chain of particles shown in Fig. $2 b$.

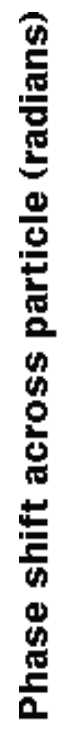

FIG. 3. Solid line: predicted phase shift vs. diameter for a uniformly magnetized $\mathrm{Fe}_{0.5} \mathrm{Ni}_{0.5}$ sphere. Results that are consistent with the prediction are shown as bold circles. Results from particles that contain 'flux tubes' are shown as open circles. 